\title{
The ten questions screen for childhood disabilities: its uses and limitations in Pakistan
}

\author{
M S Durkin, Z M Hasan, K Z Hasan
}

\begin{abstract}
Objective-To assess the accuracy of the ten questions screen as a measure of childhood disability for epidemiologic studies in populations lacking resources for professional assessment of children's development and functioning.

Design-Household survey and screening of children in phase one followed by clinical assessments in phase two.

Setting - Karachi, Pakistan.

Participants-A cluster sample of 6365 children, aged 2 to 9 years, screened using the ten questions and a subsample referred for clinical assessments.

Main results - Although the sensitivity of the ten questions as a global screen for serious cognitive, motor, and seizure disabilities is high $(84-100 \%)$, its sensitivity for identifying and distinguishing specific types of disability and for detecting vision, hearing, and mild disabilities, overall, is limited (generally $<80 \%$ and as low as $4 \%$ for mild vision disability). The predictive value of a positive screening result is also limited - using the ten questions in surveys without clinical confirmation results in overestimation of the prevalence of serious disability by more than $300 \%$.

Conclusions - The ten questions screen is not an assessment tool. Its utility lies in its ability to screen or select a fraction of the population at high risk for serious disability. As a screening tool, it allows scarce diagnostic and other professional resources to be efficiently directed toward those at high risk.
\end{abstract}

(F Epidemiol Community Health 1995;49:431-436)

G H Sergievsky

Centre, Columbia

University,

630 W. 168 St.,

New York,

NY 10032, USA

M Durkin

Department of Neuropsychiatry, Jinnah Postgraduate Medical Centre, Karachi, Pakistan Z M Hasan

Baqai Medical College, Karachi, Pakistan

K Z Hasan

Correspondence to: Dr. M Durkin.

Accepted for publication March 1995
Children in developing countries experience high incidence rates of potentially disabling conditions, including nutritional deficiencies, ${ }^{12}$ infections, ${ }^{3}$ and trauma and environmental hazards. ${ }^{45}$ Under conditions of increasing child survival, which are beginning to occur in a number of less developed countries, ${ }^{6-8}$ prevalence rates of disability are likely to rise, following patterns seen in more developed countries. ${ }^{9-11}$ Despite the public health impact of childhood disability and the need to monitor trends, there has been little epidemiologic research on this and other chronic paediatric health problems in countries that lack relevant routinely collected data. ${ }^{12}$

The ten questions screen (see Appendix) was developed as a rapid and low cost method of identifying children with serious disabilities in diverse cultures. ${ }^{1314}$ It is intended as a tool to facilitate epidemiologic research as well as referral to community based rehabilitation programmes which are being established in many places. ${ }^{15}$

Six of the ten questions (questions 1, 4, $7,8,9,10)$ are designed to detect mental retardation or serious cognitive disability. Questions 1 (delayed milestones) and 9 (impaired or delayed speech) are included among these along with questions on learning and comprehension because children with serious mental retardation typically exhibit these characteristics. ${ }^{16}$. Two questions (questions 1,5 ), including one of the mental retardation questions (question 1) are intended to detect serious motor disability. In addition, there is one question each to identify serious disabilities related to vision (question 2), hearing (question 3), and seizures (question 6).

A study in Dhaka, Bangladesh found that the ten questions screen had excellent sensitivity and high specificity for detecting serious disabilities in 2-9 year old children. It also showed that the validity was comparable for boys and girls, and for older and younger children within the 2-9 year age range. ${ }^{17}$ Investigators using the ten questions in Jamaica found that it had excellent sensitivity for serious motor, seizure, speech, vision, and hearing disabilities. ${ }^{18}$ Recent comparative analyses of the ten questions screen in three countries (Bangladesh, Jamaica, and Pakistan) found that it had good reliability (alpha and kappa coefficients greater than 0.60 ) in all three countries as well as acceptable to excellent sensitivity $(>80 \%)$ for detecting serious cognitive, motor, and seizure disabilities. It was found, however, to have relatively poor sensitivity for serious vision and hearing disorders that had not been identified previously, and it was concluded that the ten questions screen must be supplemented with actual tests of vision and hearing in order to detect these disabilities. ${ }^{1920}$ The studies in all three countries (Bangladesh, Jamaica, and Pakistan) have also shown that the screen has limited positive predictive value for serious disability $(<25 \%)$, meaning that most $(\geqslant 75 \%)$ children who screen positive do not have a serious disability. ${ }^{1619}$

All previous analyses of the ten questions screen have used a broad or global interpretation which considers a screening result to be positive for any disability if at least one question is positive; no correspondence is required between specific questions on the screen and the type of disability diagnosed. For example, a child screening positive only on question 2 for hearing who is found on clinical examination to 
have normal hearing but a moderate cognitive disability would be considered a true positive, not a false negative, in determining sensitivity, specificity, and predictive value. This global interpretation of a positive screening result is appropriate if one assumes, as the developers of the ten questions screen have done, ${ }^{1314}$ that: (a) a parent can identify that a child has a functional limitation in a general sense but should not be expected to diagnose specific disabilities; and (b) a positive screening result for any one or more questions will be followed by a clinical evaluation to confirm the presence or absence of each of the five types of disability screened for (cognitive, vision, hearing, motor, and seizure).

Despite the intended use of the ten questions as a first phase screen to be confirmed by clinical evaluation, some users of the instrument, in field settings lacking resources for clinical evaluations, have raised the question of whether it might serve as a more definitive tool than a screen. ${ }^{21}$ This application requires a disability-specific interpretation of the screening results. Under a disability-specific interpretation, a child with one type of disability would have to screen positive for that disability in order to be considered a true positive. For example, a child with a cognitive disability only, who screened positive only on the hearing question, would be classified as false negative for cognitive disability and false positive for hearing disability under a disability-specific interpretation.

This paper considers the validity of the ten questions screen when a disability-specific interpretation is applied. Specifically, it uses data from the validation study in Karachi, Pakistan to address two questions. Firstly, are responses to specific items on the ten questions screen sufficiently accurate to provide a basis for referring children to specific rehabilitation programs? Secondly, can responses to specific questions be used as a basis for estimating the prevalence of specific disabilities in the population? To answer these questions, we examine the effects on sensitivity, specificity, positive predictive value, and prevalence of applying a disability-specific compared with a global interpretation of a positive screening result.

\section{Methods}

A cluster sampling strategy modelled after that of the expanded programme on immunisation ${ }^{22}$ was used to obtain a sample of 6365 children between the ages of 2 and 9 years (inclusive) living in Greater Karachi, an area inhabited by nearly eight million people with $94 \%$ in urban and $6 \%$ in rural households. ${ }^{23} \mathrm{~A}$ two phase study design was implemented in each cluster. In phase one, a house to house survey was completed that included screening all 2 to 9 year old children for disabilities using the ten questions screen. Nearly all $(98 \cdot 7 \%)$ of the households selected in the sample were successfully contacted and agreed to participate in the study. All 2 to 9 year old children in the selected households were included in the screening. Although inclusion of more than one child per household was not optimal for statistical purposes (due to non-independence of observations), it was considered necessary for practical and ethical reasons since the screening was followed by referral for indicated assessments and treatment.

The ten questions screen (Appendix), translated into Urdu, was administered as a personal interview with a parent or guardian of the child. The child did not therefore have to be present for the screening to take place. The interviewers were social work students from Karachi, whose training and supervision was overseen by the principal investigator $(\mathrm{ZMH})$.

In phase two, all children with positive screening results and a systematic sample of about one in 12 (about $8 \%$ ) of those with negative results were referred for comprehensive clinical examinations. The clinical examinations were done without knowledge of the screening results by a team of psychologists and physicians. Standard procedures and criteria were used to make diagnoses and assess the level of disability (mild, moderate, and severe) in five areas: cognitive (mental retardation), motor, seizures, vision, and hearing. The diagnosis of mental retardation was made jointly by a psychologist and physician after they had independently examined the child and discussed their findings. The psychological assessment of mental retardation was based on non-verbal scales of the 1985 revision of the Stanford-Binet ${ }^{24}$ and an adaptive behaviour scale developed for and normed on children in Pakistan. ${ }^{25}$ Ratings of motor, seizure, vision, and hearing disabilities were based on the medical assessment. In this paper, moderate and severe disabilities are combined into a single category - serious.

All data from the household survey, the screening, and the psychological and medical evaluations were recorded on precoded forms and entered into a computerised data base. Accuracy checks and necessary corrections were made both before and after the data were entered into the data base.

The validity of the ten questions screen is evaluated against the clinical ratings of disability made for children referred to phase two of the study. Because only a sample of those screened negative was evaluated, it is necessary to compute adjusted estimates of sensitivity, specificity, and prevalence as described by Shrout and Newman. ${ }^{26}$ Thus, because only about $8 \%$ of children with negative screening results were evaluated, the data for these children were weighted by a factor of approximately 12.5 in the analysis.

Prevalence estimates per 1000 children screened (with $95 \%$ confidence intervals) are given using the global interpretation and two phase study design ${ }^{26}$ and using the disabilityspecific interpretation and a single phase design. ${ }^{27}$

Under a global interpretation of the ten questions screen, a true positive is defined as a child with a disability who was positive on any one or more of the questions. For the disabilityspecific interpretation, a child with a given 


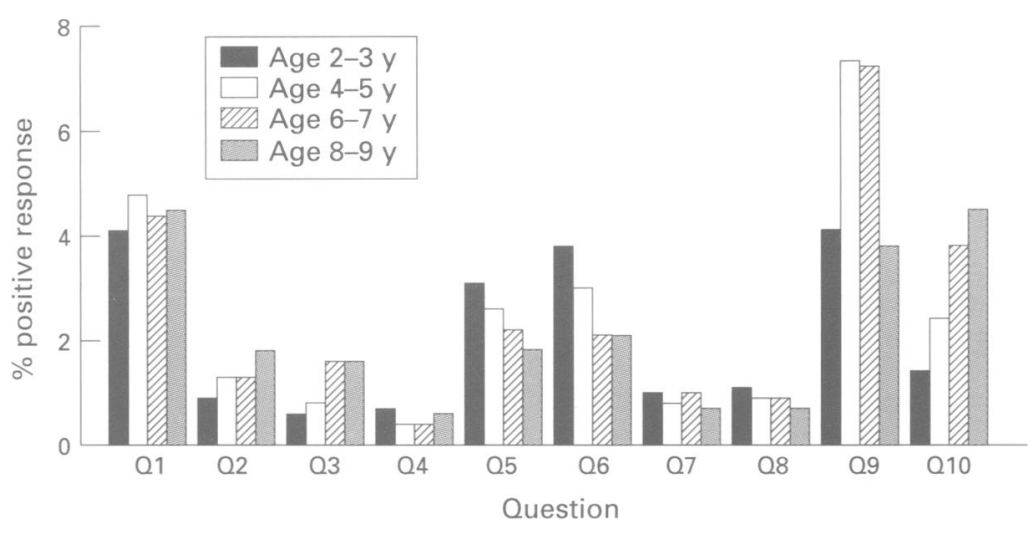

Figure Prevalence of positive responses on the ten questions screen in relation to age in Pakistani children results on the ten questions (table 1). The percentage that screened positive was somewhat higher in boys than in girls and in urban than rural areas (table 1). Questions 9 (speech) and 1 (milestones) had the highest percentages of positive responses. In rural areas, question 5 (mobiity) also elicited a high percentage of positive responses (table 1 ). The relationship between children's age and the probability of screening positive on each question is shown in the figure. The probabilities of reporting problems with vision (question 2), hearing (question 3), and slowness compared with other children (question 10) increase with age, while those for mobility (question 5) and seizures (question 6) decline with each successive age category (figure).

Under a global interpretation (table 2(A)) the sensitivity of the screen is good to excellent for serious cognitive, motor, and seizure disabilities; moderately low for serious hearing disability; and low for serious vision disability. The sensitivity is low, as expected, for all types of mild disabilities. The specificity of the screen under a global interpretation ranges from 86

\section{Results}

A total of 6365 children were screened in phase one and $936(14 \cdot 7 \%)$ had positive screening

Table 1 Ten questions screening results for children aged 2-9 years surveyed for disability in Greater Karachi

\begin{tabular}{|c|c|c|c|c|c|}
\hline & Boys & Girls & Urban & Rural & Total \\
\hline $\begin{array}{l}\text { Total no of children screened } \\
\% \text { Positive screening result }\end{array}$ & 3422 & 2943 & 5744 & 621 & 6365 \\
\hline $\begin{array}{l}\text { (any question) } \\
\% \text { Positive on question: }\end{array}$ & $16 \cdot 6$ & $12 \cdot 6$ & $14 \cdot 2$ & $19 \cdot 3$ & $14 \cdot 7$ \\
\hline 1 (cognitive, motor) & $4 \cdot 9$ & $4 \cdot 0$ & $3 \cdot 9$ & $9 \cdot 5$ & $4 \cdot 5$ \\
\hline 2 (vision) & $1 \cdot 4$ & $1 \cdot 2$ & $1 \cdot 3$ & $1 \cdot 1$ & $1 \cdot 3$ \\
\hline 3 (hearing) & $1 \cdot 2$ & $1 \cdot 1$ & $1 \cdot 1$ & 1.9 & $1 \cdot 1$ \\
\hline 4 (cognitive) & $0 \cdot 6$ & $0 \cdot 4$ & 0.5 & $0 \cdot 5$ & $0 \cdot 5$ \\
\hline 5 (motor) & $2 \cdot 6$ & $2 \cdot 3$ & $2 \cdot 1$ & $6 \cdot 0$ & $2 \cdot 5$ \\
\hline 6 (seizure) & $3 \cdot 1$ & $2 \cdot 4$ & $2 \cdot 7$ & 3.9 & $2 \cdot 8$ \\
\hline 7 (cognitive) & $1 \cdot 1$ & $0 \cdot 7$ & 0.9 & $1 \cdot 4$ & 0.9 \\
\hline 8 (cognitive, speech) & $1 \cdot 1$ & 0.7 & 0.9 & $0 \cdot 8$ & $0 \cdot 9$ \\
\hline 9 (cognitive, speech) & $6 \cdot 8$ & $4 \cdot 4$ & $5 \cdot 7$ & $6 \cdot 0$ & $5 \cdot 7$ \\
\hline 10 (cognitive) & $3 \cdot 3$ & $2 \cdot 4$ & $2 \cdot 9$ & $2 \cdot 7$ & $2 \cdot 9$ \\
\hline$\%$ Positive on $>1$ question & $4 \cdot 7$ & $3 \cdot 5$ & $3 \cdot 8$ & $7 \cdot 9$ & $4 \cdot 3$ \\
\hline $\begin{array}{l}\% \text { Of screened children who were } \\
\text { clinically evaluated }\end{array}$ & $22 \cdot 5$ & $19 \cdot 7$ & $20 \cdot 7$ & $26 \cdot 1$ & $21 \cdot 4$ \\
\hline
\end{tabular}

Table 2 Estimated sensitivity, specificity, and positive predictive value (PPV)* of the ten questions screen for cognitive, motor, seizure, hearing, and vision disabilities in Greater Karachi (2-9 year old children), by type and severity of disability, under global (part A) and disability-specific (part B) interpretations of screening results (values given are percentages).

\begin{tabular}{|c|c|c|c|c|}
\hline & \multicolumn{2}{|c|}{ (A) Global } & \multicolumn{2}{|c|}{ (B) Disability specific } \\
\hline & Serioust & Mild & Serious & Mild \\
\hline \multicolumn{5}{|l|}{ Cognitive: } \\
\hline Sensitivity & 84 & 31 & 76 & 24 \\
\hline Specificity & 87 & 86 & 91 & 91 \\
\hline PPV & - & - & 14 & 16 \\
\hline \multicolumn{5}{|l|}{ Motor: } \\
\hline Sensitivity & 84 & 32 & 75 & 20 \\
\hline Specificity & 87 & 86 & 96 & 95 \\
\hline PPV & - & - & 26 & 19 \\
\hline \multicolumn{5}{|l|}{ Seizure: } \\
\hline Sensitivity & 100 & 57 & 93 & 31 \\
\hline Specificity & 86 & 86 & 98 & 97 \\
\hline PPV & - & - & 16 & 8 \\
\hline \multicolumn{5}{|l|}{ Hearing: } \\
\hline Sensitivity & 70 & 32 & 54 & 8 \\
\hline Specificity & 86 & 86 & 99 & 99 \\
\hline PPV & - & - & 24 & 7 \\
\hline \multicolumn{5}{|l|}{ Vision: . } \\
\hline Sensitivity & 44 & 17 & 34 & 4 \\
\hline Specificity & 86 & 86 & 99 & 99 \\
\hline PPV & - & - & 39 & 21 \\
\hline \multicolumn{5}{|l|}{ Any disability: $\ddagger$} \\
\hline Sensitivity & 75 & 27 & - & - \\
\hline Specificity & 88 & 88 & - & - \\
\hline PPV & 23 & 32 & - & - \\
\hline
\end{tabular}

* Under the global interpretation, positive predictive values (PPV) are given only for the category "any disability", because a positive response in not intended to be positive for each specific disability

† Serious refers to moderate and severe levels of disability combined.

₹ The category "any disability" refers to the presence of one or more of the five specific disabilities. Validity of the ten questions for this category is relevant only under the global interpretation. 
Table 3 Estimated prevalence of 1000 (95\% confidence intervals) of cognitive, motor, seizure, vision and hearing disabilities in Greater Karachi (2-9 year old children), under global (part A) interpretation of a screening result with clinical confirmation, and under a disability-specific (part B) interpretation with no clinical confirmation

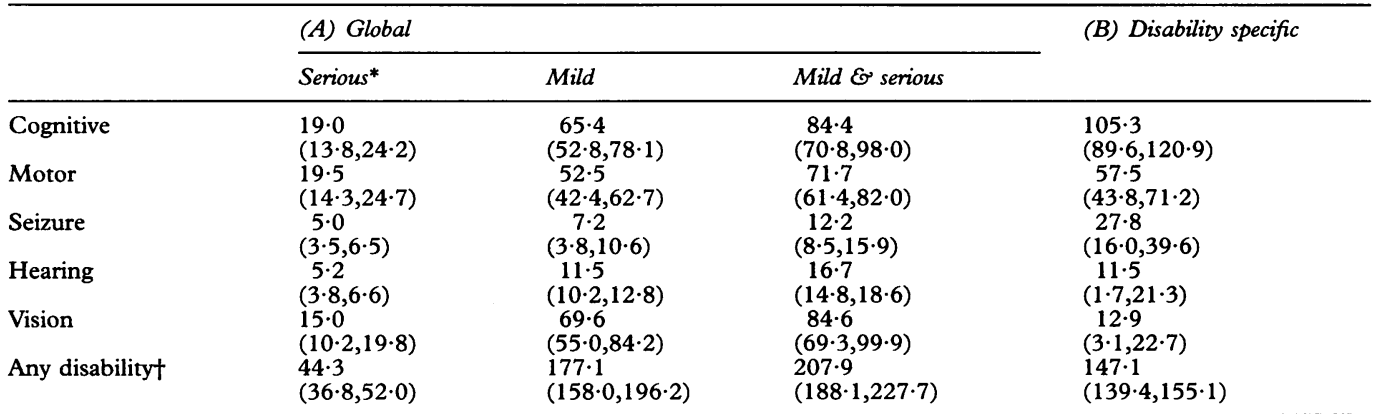

* Serious refers to moderate and severe combined.

t Under "any disability", the prevalence of mild and serious disability combined is less that the sum of mild and serious because some children have both mild and serious disabilities of different types.

to $88 \%$ for all types and levels of disability, indicating that $13-15 \%$ of children without disabilities screen positive on the ten questions when a global interpretation is used (table 2(A)). The positive predictive value for any serious disability is $23 \%$, indicating that $77 \%$ of children with positive screening results were found on clinical examination not to have a serious disability.

Using a disability-specific interpretation (table 2(B)), the sensitivity drops considerably, relative to the global interpretation, for each type of disability. Specificity, however, increases from the $86-88 \%$ range to the range of $91-99 \%$ (table 2(B)). The predictive values of the various types of positive screening results under a disability-specific interpretation range from $14 \%$ to $39 \%$ for serious grades of the respective disability types (table 2(B)).

Under both global and disability-specific interpretations, the variables maternal education, age and gender of the child, and urban-rural residence were not found to significantly affect the sensitivity of the screen. This was investigated in logistic regression analyses, which showed these variables did not significantly affect the odds of a false negative screening result among children with serious disabilities.

Estimated prevalence rates per 1000 children (with $95 \%$ confidence intervals) of each type of disability are given in table 3 based on a global interpretation followed by clinical confirmation (part A) and based on a disabilityspecific interpretation with no clinical confirmation (part B). Part A gives our best estimate of the true prevalence in the population, and part $B$ gives estimates that would be obtained if the screen were used with no clinical confirmation in phase two. For serious cognitive, motor, seizure, and hearing disabilities, the use of a disability-specific interpretation with no clinical confirmation results in substantially inflated estimates of prevalence. For serious vision disability, the disability-specific approach results in a small underestimate. For any serious disability, the disability-specific approach overstates prevalence by more than $300 \%$ (147.1 $v 44.3$ per 1000 , table 3$)$.

When the prevalence of disability regardless of severity (mild and serious) is considered under a disability-specific interpretation, pre- valence is much less inflated for cognitive and seizure disabilities, somewhat underestimated for motor and hearing disabilities, and very underestimated for vision disability (table 3 ).

\section{Discussion}

Achieving maximum sensitivity is a priority if the purpose of screening is to cast a broad net, identifying a subgroup of the population that includes all or nearly all with serious disability. Given this purpose and the availability of professional or other diagnostic resources to provide confirmation in phase two, the higher sensitivity obtained under a global interpretation is preferred to the higher specificity associated with a disability-specific interpretation of the ten questions.

Maximum specificity would be a priority if the purpose were to identify children likely to have a serious specific disability for direct referral to rehabilitation services in settings where professional resources are unavailable to confirm the screening result. Under these conditions, the disability-specific interpretation is preferable because it insures that fewer than $10 \%$ of children without disability will screen positive (that is, specificity $>90 \%$ ). Specificity, however, is not a sufficient criterion if the intention is to use the ten questions screen as a basis for referral to rehabilitation services. The limited predictive values of positive screening results, even under a disability-specific interpretation, suggest that well over half of the children who screen positive will be false positives for serious disability. Thus, clinical confirmation of positive screening results is needed to distinguish true from false positive test before referral to services. In settings where second phase clinical evaluations can be arranged to confirm the screening results, the global interpretation of the ten questions is therefore, clearly the preferred approach because of its higher sensitivity.

Although the clinical examinations are costly and require a level of professional resources not available in all communities, these examinations are an essential component of screening programmes and serve two important functions in addition to validating the screen. One is to provide information on the diagnosis 
and possibly the cause of disability - information that can be used to guide public health prevention as well as individual treatment efforts. Another is to provide a plan for rehabilitation and referral to services. Thus, this research suggests that the development of appropriate screening procedures, though useful and essential, is not enough. Communities also require minimal levels of professional resources to provide validation of screening results as well as diagnostic and clinical information to serve as a basis for rehabilitation, referral and public health interventions.

The validation results show that for mild disabilities overall and for serious grades of vision and probably hearing disability, the ten questions does not provide a sufficiently accurate screen. Regardless of whether a global or disability-specific approach is used, sensitivity is too low to be useful for identifying these disabilities. Thus, for mild disabilities, an approach other than the ten questions, perhaps one that involves direct testing of children, is required. Since many rehabilitation techniques are particularly beneficial for children with mild levels of disability, the development of low cost and cross culturally appropriate screening procedures for mild disability should be viewed as a priority for future work. Similarly, for detection of all grades of vision and hearing loss that have not been previously identified, the questionnaire approach has limited sensitivity, suggesting that direct testing of vision and hearing should be employed.

After observing that the ten questions screen lacks sensitivity for serious vision and hearing disabilities not previously identified, we considered what the effect would be on the sensitivity of the screen for cognitive, motor, and seizure disabilities, if questions 2 (vision) and 3 (hearing) were dropped from the questionnaire. Using only the eight remaining questions, the sensitivity under a global interpretation is essentially unchanged for serious cognitive, motor, and seizure disabilities (one child with serious cognitive disability classified as true positive under a global interpretation was positive only on question 3 for hearing disability, causing a decline in sensitivity for cognitive disability from $84 \%$ to $83 \%$ when questions 2 and 3 are omitted). This result suggests future work should confirm the utility of including questions 2 and 3, particularly if the questionnaire is to be supplemented with tests of vision and hearing. Although the ten questions does seem sensitive for these disabilities in populations where children with serious vision and hearing disabilities have been previously identified and brought to the attention of parents, ${ }^{19}$ the primary utility of the ten questions lies in its ability to identify children with disabilities not previously identified and treated. For this purpose, the questionnaire approach seems to lack utility for screening of vision and hearing disability.

Though low enough to require clinical confirmation, the predictive value of a positive ten questions screening result for any disability is, nonetheless, substantial. This value in Karachi for any serious disability under a global interpretation is $23 \%$, implying that the prevalence of serious disability among children who screen positive is 230 per 1000 , a value that is more than five times the estimated prevalence of serious disability in the population (44.3 per 1000 , table 3$)$. Thus, the ten questions is effective in identifying a subpopulation in which the prevalence of serious disability is high. Although professional resources are required to confirm a positive result, the screen enhances the efficiency of those resources. To identify approximately $90 \%$ of children with serious cognitive, motor or seizure disabilities, health professionals in Karachi would need to evaluate not all children but only the fraction $(14 \cdot 7 \%)$ who screen positive on the ten questions.

The prevalence estimates clearly show that the disability-specific interpretation with no clinical confirmation provides little correspondence to the true prevalence of disability in the population. For serious disability overall, and for each type other than vision, the screen overestimates prevalence. This finding has important implications for census and survey data on the prevalence of disability in populations. Typically, such data are obtained from a single question on the national census or from a series of survey questions. ${ }^{28}$ Experience with the ten questions suggests that this approach, which is widely used to generate national and international statistics on disability, ${ }^{29}$ may overestimate the prevalence of serious disabilities and underestimate the prevalence of mild and serious disabilities combined.

In conclusion, we have shown that the ten questions is useful as a screen for serious cognitive, motor, and seizure disabilities in 2 to 9 year old children. It is not sufficiently accurate, however, to provide an ultimate measure of disability or of the frequency of serious childhood disabilities in populations. Confirmation of positive screening results is required to distinguish true from false positive results. In the present study, confirmation of both positive and a sample of negative screening results was provided by a team of professionals with expertise in pediatrics, neurology and psychology. Future work is required to determine whether lower cost and more broadly feasible methods can be developed to confirm a positive ten questions screen.

We have also shown that for vision and hearing disabilities, and for mild disabilities overall, low cost screening methods with greater sensitivity than the ten questions must be developed for use in less developed countries to monitor prevalence and to identify children who might benefit from rehabilitation services. Like the ten questions, these methods should aim to enhance the efficiency of scarce professional resources and attain validity within contexts of limited resources and diverse cultural and socioeconomic conditions.

This work was supported by the BOSTID Program of the National Academy of Sciences (USA), the Epilepsy Foundation of America, the National Institute of Neurological Diseases and of America, the National Institute of Neurological Diseases and Stroke (R29 NS27971), and the New York State Psychiatric Institute. The authors would like to acknowledge the contributions of Drs Zena Stein, Lillian Belmont, Marigold Thor- 
burn, Sultana Zaman, Leslie Davidson and Patrick Shrout to the design and conduct of this research.

1 Hetzel BS, Potter BJ, Dulberg EM. The iodine deficiency disorders: nature, pathogenesis and epidemiology. World Review of Nutrition and Dietetics 1990;62:59-119.

2 Brown KH, Solomons NW. Nutritional problems of developing countries. Infect Dis Clin North Am 1991;5(2) 297-317.

3 Yunes J, Chelala C, Blaistein N. Children's health in developing countries: much remains to be done. World Health Forum, 1994;15(1):73-76.

4 Smith GS, Bares P. Unintentional injuries in developing countries: the epidemiology of a neglected problem. Epidemiol Rev 1991;13:228-66.

5 Richman N. After the flood. Am f Public Health 1993; 83(11):1522-24

6 Ewbank DC. Impact of health programmes on child mortality in Africa: evidence from Zaire and Liberia. Int $f$ Epidemiol 1933;22(suppl 1):S64-72.

7 Bahr J, Wehrhahn R. Life expectancy and infant mortality in Latin America. Soc Sci Med 1993;36(10):1373-82.

8 Pison G, Trape JF, Lefebvre M, Enel C. Rapid decline in child mortality in rural areas of Senegal. Int $\mathcal{f}$ Epidemio 1993;22(1):72-80.

9 Hagberg B, Hagberg G, Olow I. The changing panorama of cerebral palsy in Sweden IV: epidemiological trends 1959-1978. Acta Paediatr Scand 1984;73:433-40.

10 Stanley FJ, Watson L. Trends in perinatal mortality and cerebral palsy in Western Australia. BMF 1992;304(6843): 1658-63.

11 Bushan V, Paneth N, Kiely JL. Impact of improved survival of very low birth weight infants on recent secular trends in the prevalence of cerebral palsy. Pediatrics 1993;91(6): 1094-2000.

12 Stein Z, Susser M. The less developed world: Southeast Asia as a paradigm. In: I Wortis ed. Mental retardation and developmental disabilities: an annual review, XI, New York: developmental disabilities: an ann

13 Belmont L. Screening for severe mental retardation in developing countries: the International Pilot Study of Severe Childhood Disability. In: Berg JM ed. Science and technology in mental retardation. London: Methuen, 1986;38995.

14 Stein ZA, Durkin MS, Davidson LL, Hasan ZM, Thorburn MJ, Zaman SS. Guidelines for identifying children with mental retardation in community settings, In: World Health Organization. Assessment of people with mental retandation Geneva: World Health Organization, 1992;1241.

15 Helander E, Mendis P, Nelson G, Goerdt A. Training in the community for people with disabilities. Geneva: World Health Organization, 1989.

16 Grossman HJ, ed. Classification in mental retardation. Washington, DC: American Association on Mental Deficiency, ington,

17 Zaman S, Khan N, Islam S, Banu S, Dixit S, Shrout P, Durkin $M$. Validity of the ten questions for screening serious childhood disability: results from urban Bangladesh. International $\mathcal{}$ Epidemiol, 1990;19(3):613-20.

18 Thorburn MJ, Desai P, Paul TJ, Malcolm L, Durkin MS Davidson LL. Identification of childhood disability in Jamaica: the ten question screen. Int $\mathcal{F}$ Rehabil Res 1992 15:115-27.

19 Durkin MS, Davidson LL, Desai P, et al. Validity of the ten questions screen for childhood disability: results from population-based studies in Bangladesh, Jamaica and Pakistan. Epidemiology, 1994;5:283-9.

20 Durkin MS, Wang W, Shrout PE, Zaman SS, Hasan ZM, Desai P, Davidson LL. Evaluating a ten questions screen in different cultures. $f$ Clin Epidemiol 1995;48:657-66.

21 Natale JE, Joseph JG, Bergen R, et al. Prevalence of childhood disability in a southern Indian city: independent effect of small differences in social status. Int $\mathfrak{f}$ Epidemiol 1992;21 (2):367-72.

22 Lemeshow S, Stroh G. Sampling techniques for evaluatin health parameters in developing countries, Washington, DC: National Academy Press, 1988.
23 Bureau of Statistics, Government of Sind, Pakistan. De velopment Statistics of Sind, 1986. Karachi: Planning an Development Department, Bureau of Sind, Government of Sind, 1986 .

24 Thorndike RM, Hagen EP, Sattler JM. Standford-Binet intelligence scales 4th ed. Riverside, CA: Riverside Publishing Co, 1986.

25 Hasan ZM. Problems with the use of western-style tests to assess cognitive disability in Pakistan. In: Proceedings of the International Association for the Scientific Study of Mental Deficiency eighth world congress, 1988.

26 Shrout $P$, Newman $S$. Design of two-phase prevalence surveys of rare disorders, Biometrics, 1989;45:549-55.

27 Fleiss JL. Statistical methods for rates and proportions. 2nd ed. New York: John Wiley and Sons, 1981 .

28 Chamie $M$. Survey design strategies for the study of disability. World Health Stat $Q \quad 1989 ; 42(3): 122-40$.
a

29 United Nations. Disability statistics compendium. New York: United Nations Statistical Office, 1990. ST/ESA/STAT/ SER.Y/4.

\section{Appendix}

THE TEN QUESTIONS

1 Compared with other children, did the child have any serious delay in sitting, standing or walking?

2 Compared with other children does the child have difficulty seeing, either in the daytime or at night?

3 Does the child appear to have difficulty hearing?

4 When you tell the child to do something, does he/she seem to understand what you are saying?

5 Does the child have difficulty in walking or moving his/her arms or does he/she have weakness and/or stiffness in the arms or legs?

6 Does the child sometimes have fits, become rigid, or lose consciousness?

7 Does the child learn to do things like other children his/her age?

8 Does the child speak at all (can he/she make himself/herself understood in words; can he/she say any recognizable words)?

9 For 3 to 9 year olds ask: Is the child's speech in any way different from normal (not clear enough to be understood by people other than his/her immediate family)?

For 2 year olds ask:

Can he/she name at least one object (for example, an animal, a toy, a cup, a spoon)?

10 Compared with other children of his/her age, does the child appear in any way mentally backward, dull or slow? 